\title{
Deformation Behavior of Pure Titanium With a Rare HCP/FCC Boundary: An Atomistic Study
}

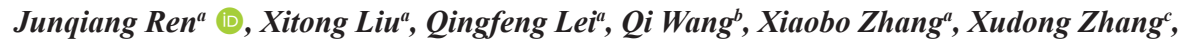 \\ Xuefeng Lü*,Hongtao Xue ${ }^{a}$, Yutian Ding ${ }^{a}$ \\ a State Key Laboratory of Advanced Processing and Recycling of Nonferrous Metals, Department of \\ Materials Science and Engineering, Lanzhou University of Technology, Lanzhou 730050, PR China \\ ${ }^{b}$ Huanghuai University, Zhumadian, Henan 463000, PR China \\ ${ }^{c}$ Center for High Performance Computing, Network Information Center, Xi' an Jiaotong University, \\ Xi'an 710049, PR China
}

Received: November 21, 2019; Revised: January 22, 2020; Accepted: March 01, 2020

\begin{abstract}
The compressive and tensile behaviors in a Ti nanopillar with a biphasic hexagonal close-packed (HCP) /face-centered cubic (FCC) phase boundary are theoretically researched using classic molecular dynamic simulation. The results indicate that the HCP/FCC interface and free surface of the nanopillar are the sources of dislocation nucleation. The plastic deformation is mainly concentrated in the metastable FCC phase of the biphasic nanopillar. Under compressive loading, a reverse phase transformation of FCC to the HCP phase is induced by the dislocation glide of multiple Shockley partial dislocations $\frac{1}{6}<\overline{1} 21>$ under compressive loading. However, for tensile loading a large number of Lomer-Cottrell sessile dislocations and stacking fault nets are formed when the partial dislocations react, which leads to an increase in stress. The formation mechanism of a Lomer-Cottrell sessile dislocation is also studied in detail. Shockley partial dislocations are the dominant mode of plastic deformation behaviors in the metastable FCC phase of the biphasic nanopillar.
\end{abstract}

Keywords: Molecular dynamics simulation, Titanium, Dislocations, Biphasic nanopillar, Phase transformations.

\section{Introduction}

$\mathrm{Ti}$ and its alloys are used in a wide variety of engineering sectors, including aerospace, automotive, scientific instrumentation, chemical engineering and bioengineering due to their high strength and toughness, excellent resistance to fatigue and corrosion, low thermal conductivity, high temperature performance and biocompatibility ${ }^{1}$. Pure Ti and its majority alloys exhibit a hexagonal close-packed (HCP) crystal structure ( $\alpha$-phase) at room temperature and a body-centered cubic structure ( $\beta$-phase) above the transus temperature $\left(>882 \pm 2^{\circ} \mathrm{C}\right)^{2}$. Hydrostatic pressure, shock loading or high-pressure torsion processing can induce transformation from the $\mathrm{HCP} \alpha$-phase to hexagonal $\omega$-phase in $\alpha-\mathrm{Ti}^{3-6}$. A transformation from the $\omega$-phase to the orthorhombic $\gamma$-phase was observed in Ti under an external pressure of $116 \mathrm{GPa}^{7}$.

In addition to these typical structures, the possible occurrence of a phase with a face-centered cubic (FCC) crystal structure has been widely observed in Ti and its alloys, which is phase that does not exist in the conventional pressure-temperature phase diagrams of Ti. Experimental observations showed that the presence of the FCC phase was associated with thin films, interfaces, powders, high levels of plastic deformation, and heat-treated $\mathrm{Ti}$ alloys. Recent work shows that the small FCC precipitates formed

*e-mail: luxuefeng1979@163.com in freestanding thin foils during in situ transmission electron microscope (TEM) heating ${ }^{8}$. Experimental results indicate that the stability of FCC Ti in thin films is related to the film thickness, surface orientation, and temperature ${ }^{9-14}$. The HCP to FCC phase transformation in $\mathrm{Ti}$ is dominated by the high density of dislocations and twins introduced by ball milling but not stacking faults ${ }^{15}$. Another experiment observed small amounts of FCC Ti after ball milling Ti-Al powders. The transformation was induced thermally by the energy provided from the ball milling process ${ }^{16}$. The formation of FCC Ti in heat-treated Ti-6Al-4V alloy caused by adding $\mathrm{Zr}$, which can decrease the basal stacking fault energy ${ }^{17}$. The tress-induced HCP to FCC phase transformation was attributed to the gliding of Shockley partial dislocations ${ }^{18}$. First principles calculations and Gibbs free energy calculation demonstrated the possible occurrence of an FCC phase in pure $\mathrm{Ti}^{19-21}$. Gibbs free energy calculations showed that the Gibbs free energy decreases with the shape factor and increases with decreasing particle size ${ }^{18}$. Therefore, HCP to FCC phase transformation not only exists in bulk material but also occurs on the nanometer scale. This prediction is consistent with the experimental result of the presence of FCC phase in thin films or under high strains, which has been explained by the minimization of Gibbs free energy. In our previous work ${ }^{22}$, the phase transformation in the $\{10 \overline{1} 2\}<10 \overline{1} 1>$ twinning region was also predicted in $\alpha-\mathrm{T} i$ 
single-crystal nanopillars by molecular dynamics (MD) simulations. This rare phase transformation is induced by the dislocation glide of multiple Shockley partial dislocations under the condition of size restriction and is strongly affected by temperature and strain rate ${ }^{23}$.

The stress-induced HCP to FCC phase transformation was related to the gliding of Shockley partial dislocations, and the orientation relation between the two phases has been characterized as $\left.\left.<0001\rangle_{\mathrm{HCP}} \|<001\right\rangle_{\mathrm{FCC}},\langle 10 \overline{1} 0\rangle_{\mathrm{HCP}}||<1 \overline{1} 0\right\rangle_{\mathrm{FCC}}$ and $\left.\langle 11 \overline{2} 0\rangle_{\text {HCP }}||<110\right\rangle_{\mathrm{FCC}^{17}}$, The mechanical behavior and deformation mechanism of commercial pure titanium foils and a detailed mechanism of phase transformation with orientation are proposed, to including the three stages of slip, adjustment and expansion by means of first principles calculations and experiments ${ }^{24}$. Different from the phase transition mechanism caused by the gliding of Shockley partial dislocations, the nucleation of FCC-Ti is accomplished via a pure-shuffle mechanism and the growth via shear-shuffle mechanis was studied by combining transmission electron microscopy and density functional theory calculations $^{23}$. The orientation relation of FCC-Ti and HCP-Ti are $\langle 0001\rangle_{\mathrm{HCP}} \|\langle 001\rangle_{\mathrm{FCC}}$ and $\{1010\}_{\mathrm{HCP}} \|\{110\}_{\mathrm{FCC}}$. Both two distinctive orientation relations between HCP phase and FCC phase were observed simultaneously in cold-rolled pure $\mathrm{Zr}^{25}$.

It has been widely accepted that sample size may exert a significant influence on the mechanical properties and deformation mechanism of materials on the micron- and submicron scales. In addition, the HCP to FCC phase transformation which does not exist in bulk material may occur on nanometer scale ${ }^{18}$. This prediction is consistent with experimental result that Ti with an FCC structure is formed under ambient conditions ${ }^{10,22}$. Therefore, a nanopillar model with free boundary conditions was established in this work to study the deformation behavior of pure titanium with a rare HCP/FCC boundary. Although many experimental and simulation works have demonstrated the HCP to FCC phase transformation in pure $\mathrm{Ti}$ at ambient temperature under extreme pressure, temperature, high levels of plastic deformation and size restriction conditions, the effect of FCC phase on the deformation mechanism of Ti is not clear thus far. By means of MD calculations, the present study is dedicated to proposing a detailed deformation behavior for Ti nanopillars containing an $\mathrm{HCP} / \mathrm{FCC}$ phase boundary with the orientation relation of $<0001>_{\mathrm{HCP}}\left\|<001>_{\mathrm{FCC}},<10 \overline{1} 0>_{\mathrm{HCP}}\right\|<1 \overline{10}>_{\mathrm{FCC}}$ and $<11 \overline{2} 0>_{\text {HCP }}||<110>_{\text {FCC }}$. In addition, the stability of the FCC phase has been systematically studied.

\section{Simulation Model and Method}

The schematic of bicrystal Ti with an HCP/FCC phase boundary under compression and tension loading conditions is depicted in Figure 1. The initial configuration of the HCP/FCC bicrystal consists of approximately 201933 atoms and the dimensions of the samples in $\mathrm{X}-, \mathrm{Y}-$ and $\mathrm{Z}$ - are $12 \mathrm{~nm}, 24 \mathrm{~nm}$ and $12 \mathrm{~nm}$, respectively. Free boundary conditions are applied in the $\mathrm{x}, \mathrm{y}$ and $\mathrm{z}$ directions to simulate the configuration of nanocrystal. The crystallographic orientations of the HCP phase and the FCC phase are as follows: $\mathrm{x}:<2 \overline{1} \overline{1} 0>_{\mathrm{HCP}} \mid<011>_{\mathrm{FCC}}, \mathrm{y}$ : $<01 \overline{1} 0>_{\text {HCP }}||<0 \overline{1} 1>_{\mathrm{FCC}}$, and z: $<0001>_{\text {HCP }} \|<100>_{\mathrm{FCC}}$, as shown in Figure 1a,b. In the HCP phase, the crystal structure has a known lattice constants of $\mathrm{a}=0.2951 \mathrm{~nm}$ and $\mathrm{c}=0.4683 \mathrm{~nm}$ $(\mathrm{c} / \mathrm{a}=1.587)$, whereas in the FCC phase, $\mathrm{a}=0.4174 \mathrm{~nm}$, corresponding to the minimum cohesive energy $\left(\mathrm{E}_{\mathrm{coh}}=-4.839 \mathrm{eV}\right)$. All the MD simulations are performed with a time step of 1.0 fs. The conjugate gradient (CG) algorithm is used for energy minimization to obtain equilibrium configurations before uniaxial tensile (or compressive) loading. Then, the Nosé-Hoover thermostat ${ }^{26,27}$ is used to further equilibrate the Ti bicrystal systems. The canonical ensemble(NVT), i.e., constant atom number( $\mathrm{N})$, volume $(\mathrm{V})$, and temperature(T), is applied to keep the system temperature at a constant $300 \mathrm{~K}$. The relaxed
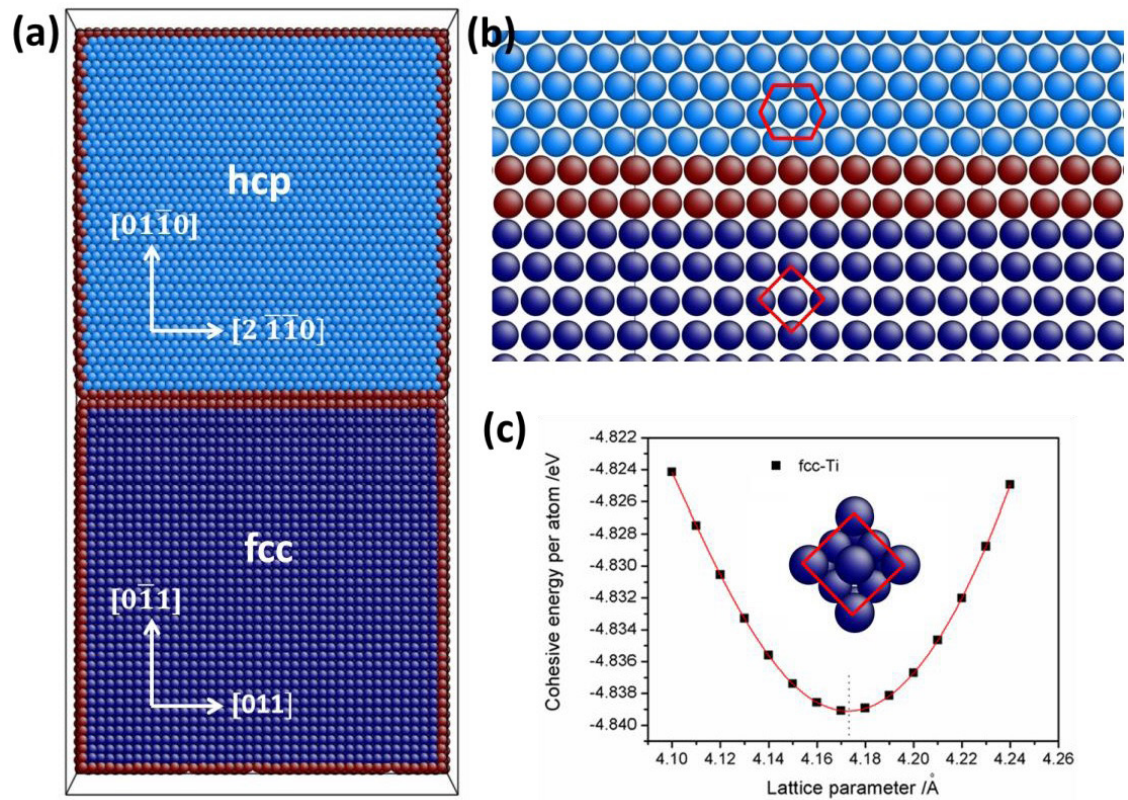

Figure 1 - (a) Setup of the simulation bicrystal. (b) Cross sectional view of the HCP-FCC interface. The hexagon and square show the HCP phase and FCC phase, respectively. (c) The FCC phase equilibrium lattice constant is obtained. 
bicrystal nanopilars are then loaded along the y-direction, with the atoms at the ends fixed to replicate loading grips. The strain rate during loading is $1 \times 10^{8} \mathrm{~s}^{-1}$. The MD calculations were carried out using the large-scale parallel molecular dynamics program LAMMPS ${ }^{28}$ and visualized with the visualization program AtomEye ${ }^{29}$. Common neighbor analysis $(\mathrm{CNA})^{30,31}$ is used to distinguish a variety of defects and the simple local lattice structure. The CNA pattern is a useful measure of the local crystal structure around an atom in the simulation system. There are five kinds of CNA patterns that LAMMPS recognizes $(\mathrm{FCC}=1, \mathrm{HCP}=2, \mathrm{BCC}=3$, icosahedral $=4$, and unknown $=5$ ). The CNA calculation can be sensitive to the specified cutoff value, and the following formula can be used to obtain a good cutoff distance:

$$
\mathrm{r}_{\mathrm{c}}^{\mathrm{hcp}}=\frac{1}{2}\left(1+\sqrt{\frac{4+2 \mathrm{x}^{2}}{3}}\right) \mathrm{a}
$$

where $\mathrm{a}=2.951$ is the lattice constant for the crystal structure concerned and in HCP-Ti, $\mathrm{x}=(\mathrm{c} / \mathrm{a})=1.587$, where 1.587 is the ideal c/a for HCP-Ti crystals.

The Finnis-Sinclair many-body potential in the MD simulation was developed by Ackland ${ }^{32}$, which is adopted to describe Ti-Ti ineratomic interactions in the simulation models. The Finnis-Sinclair many-body potential function can be expressed by

$$
E_{i}=\frac{1}{2} \sum_{j} V\left(r_{i j}\right)-\left[\sum_{j} \varphi\left(r_{i j}\right)\right]^{1 / 2}
$$

where $V$ is a pairwise function between neighbouring atoms $i$ and $j . \phi$ is the electronic density function. $\mathrm{r}_{\mathrm{ij}}$ is the distance between atom $i$ and one of the neighbouring atoms $\mathrm{j}$. The potential has been used extensively in various MD studies, including those for Ti single crystals ${ }^{33-37}$ and nanocrystals $^{38}$. The transition of the FCC phase to the HCP phase in a pure Ti nanopillar was successfully observed using this potential function under applied stress ${ }^{21}$. In addition, the effect of temperature and strain rate on the HCP-FCC phase transition was also successfully simulated by using the potential ${ }^{22}$. The lowest energy state of the FCC phase exists when the lattice constant is $0.4174 \mathrm{~nm}$.

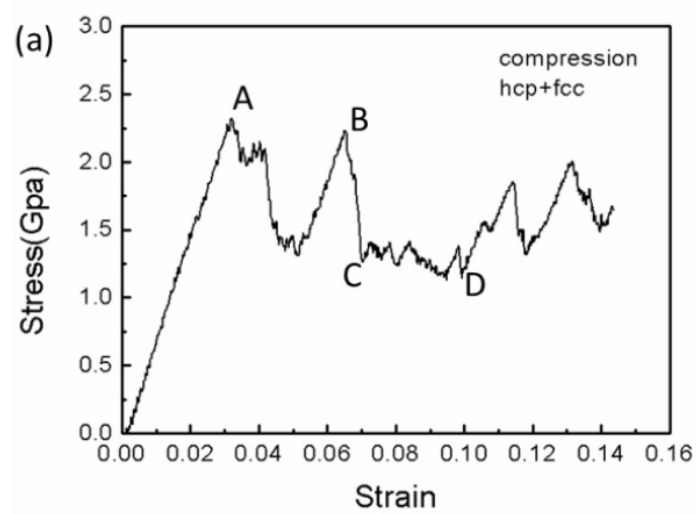

\section{Results}

\subsection{Mechanical response of the bicrystal nanopillars under uniaxial compression and tension}

The stress-strain curves of a Ti bicrystal nanopillar oriented in the $[01 \overline{1} 0]_{\mathrm{HCP}} \|[0 \overline{1} 1]_{\mathrm{FCC}}$ direction subjected to tension and compression are shown in Figure 2. The nonapillars under different loading directions show a similar maximum stress $2.32 \mathrm{GPa}$ (tagged points $\mathrm{A}$ and $\mathrm{C}$ in Figure 2a and Figure $2 b$, respectively). The yield stress is the maximum stress at a strain of $3.178 \%$ under compressive load. As shown in Figure 1a, after the initial yield peak, the flow stress of the bicrystal nanopillars builds up again to a second stress peak at point B. After the second stress peak, further loading causes an extensive plateau with a constant stress of approximately $1.25 \mathrm{GPa}$ at strain $7 \% \sim 10 \%$ (Figure 1a). However, two stress drops have occurred before the stress reaches its maximum under tensile load, at points $\mathrm{A}$ and $\mathrm{B}$ in Figure 2b, respectively. The maximum stress at a strain of $3.632 \%$ (point $\mathrm{C}$ in Figure $2 \mathrm{~b}$ ) is no longer the yield stress. The yield point occurs at a strain of $0.55 \%$.

\subsection{Deformation mechanism under compression and tension}

To better understand the different plasticity behaviors, the substructure variation and dislocation evolution during compression are carefully studied. The dislocation evolution is illustrated in Figure 3a-d, which corresponds to point A in the stress-strain curves in Figure 2. In this and subsequent figures, the atoms are colored according to CNA. The HCP atoms are colored light blue, the FCC atoms are colored dark blue, and the defect and surface atoms are colored red. For clarity, the atoms on the perfect FCC lattice are not shown in Figure 3a-d. As shown in Figure 3a-d, under compression loading, partial dislocations nucleate and grow into dislocation lines at the interface of the HCP-phase and FCC-phase of the nanopillar where stress concentration occurs as plastic deformation progresses.

The Burgers vector was determined to $b e \mathbf{b}_{1}=\frac{1}{6}[1 \overline{12}]$ on the basis of crystallographic analysis, which indicates that

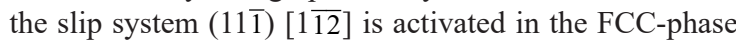
under compression. The partial dislocation glides along

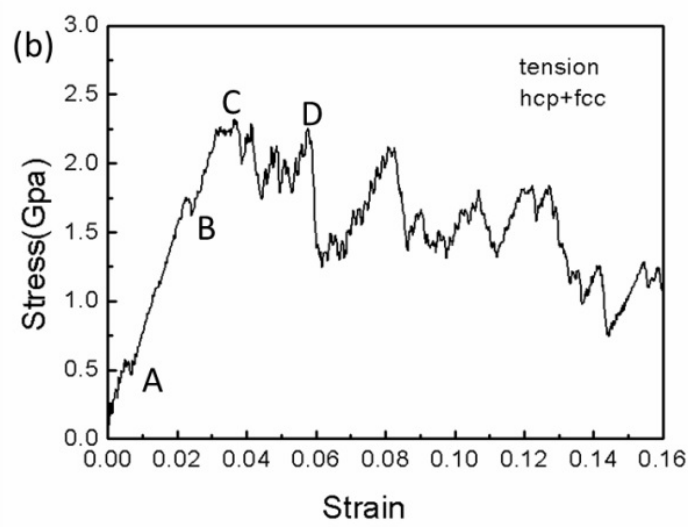

Figure 2 - Stress-strain curves for bicrystal nanopilars under compression and tension. 
(a)

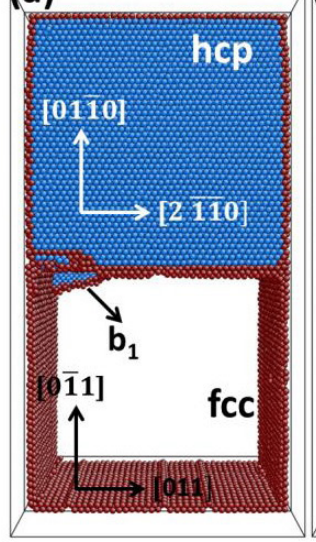

(b)

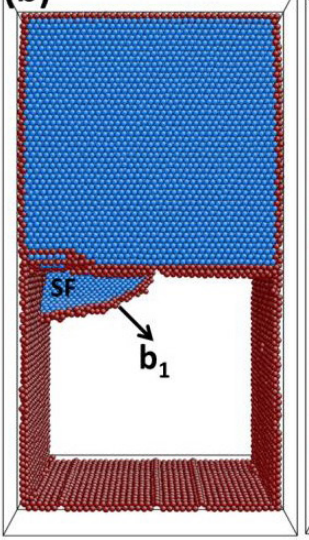

(c)

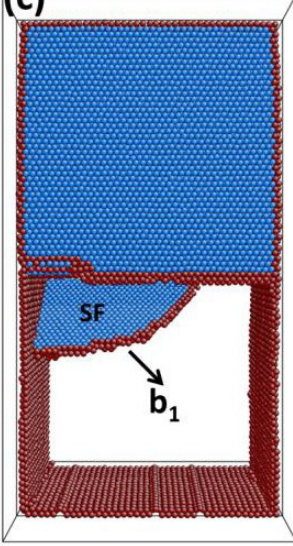

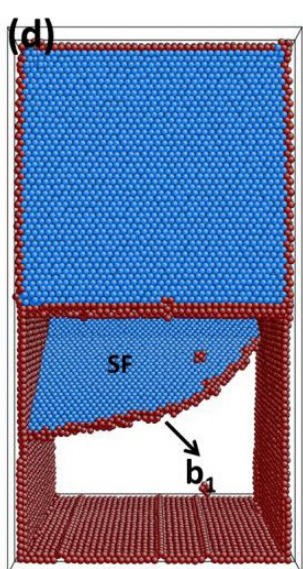

Figure 3 - Snapshots of Ti bicrystal nanopillars under compressive loading. $\mathbf{b}_{\mathbf{1}}$ is the Burgers vector of the partial dislocation. The partial dislocation with Burgers vector $\mathbf{b}_{\mathbf{1}}$ is indicated by a black arrow in snapshots.

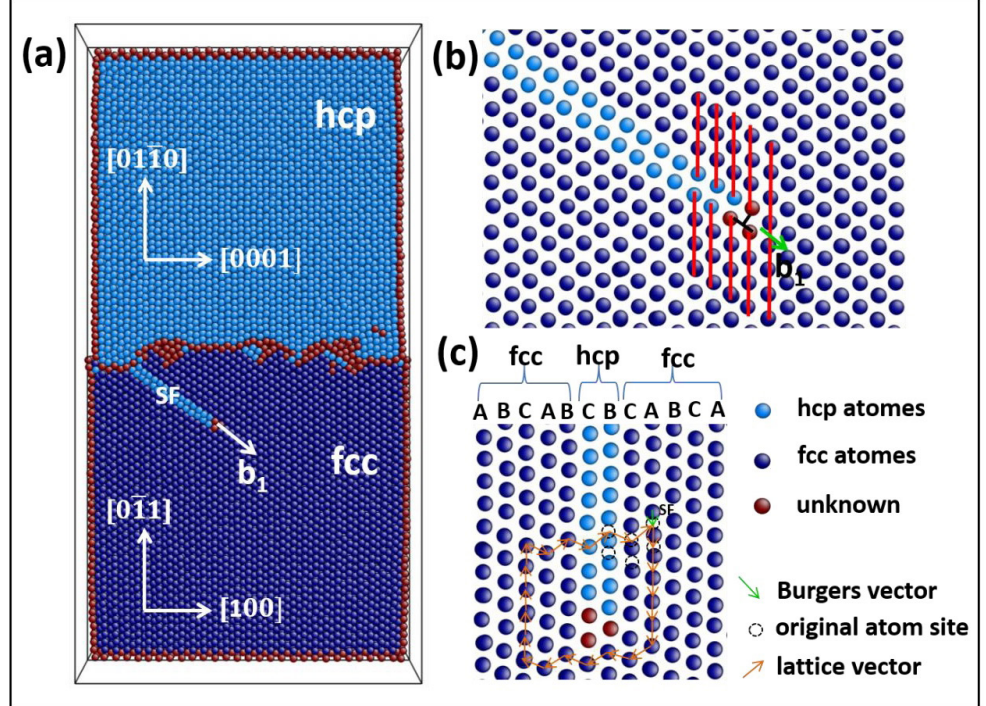

Figure 4 - The partial dislocation and stacking fault found in Figure 3. (a) Sectional view along the [2 $\overline{1} \overline{1} 0]$ of the HCP-phase or [011] of the FCC-phase in the nanopillar. (b) Atomic arrangements of the stacking fault in a plane representation. (c) SF with partial dislocation of $\mathbf{b}_{1}=\frac{1}{6}[1 \overline{12}]$

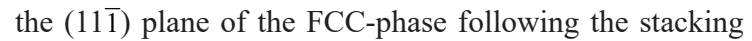
faults (SF), which are marked by the black capital letter "SF" in Figure 3b-d.

Figure 4 a shows the partial dislocations and SFs found in Figure 3. The stacking sequence of $\{111\}$ planes is perfect outside the partial dislocations (ABCABC...). The SFs formed in the nanopillar can be identified as shown in Figure 4b-c, and the SFs correspond to ABCBCABC.... All the partial dislocations and stacking faults are characterized in the conventional manner with a right handed Burgers circuit in Figure $4^{38}$. Furthermore, the partial dislocation is determined to be an edge-type Shockley partial dislocation because the dislocation is perpendicular to the Burgers vectors $\mathbf{b}_{1}=\frac{1}{6}[1 \overline{12}]$. These edge partial dislocation lines are initiated and propagated along the slip plane (111) and ultimately disappeared from the nanopillar surfaces. With further compression, the deformation of the nanopillar is primarily controlled by the partial dislocations, as marked by the symbol " $\perp$ " in Figure $4 \mathrm{~b}$. The continuous accumulation of a high density of deformation faults was caused by the dislocation glide of multiple Shockley partial dislocations, eventually leading to the phase transformation from FCC to HCP, and surface steps are left (Figure 5a). There is no plastic deformation in the HCP phase. As the strain increases further, a partial dislocation with Burgers vector $\mathbf{b}_{1}=[1 \overline{12}]$ on the slip plane (111) nucleates from the corner of the nanopillar at a strain level of $5.6 \%$. The partial dislocation is blocked by the new HCP phase, as indicated by white dotted lines in Figure 6. This site becomes a new dislocation source, and the Shockley partial dislocation is nucleated here. Another partial dislocation with Burgers vector $\mathbf{b}_{2}=\frac{1}{6}[\overline{1} 21]$ nucleates at the free surface or corner and propagates along the (11) plane.

Figure 7a-d shows snapshots of the configurations at different tension strains. In contrast to the compression process, the 

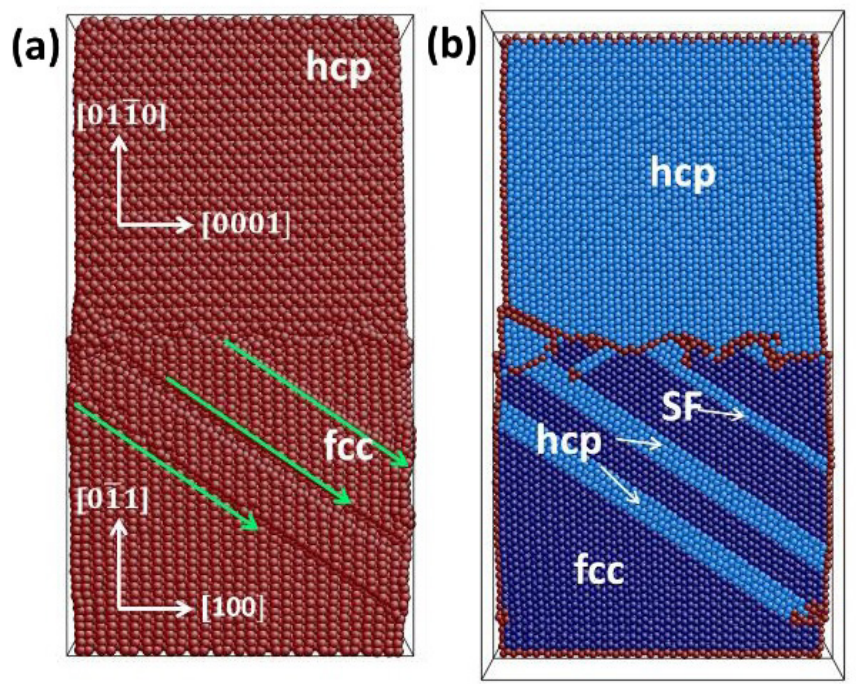

Figure 5 - Transformation from FCC phase to HCP phase in the FCC phase of the nanopillar under compression.

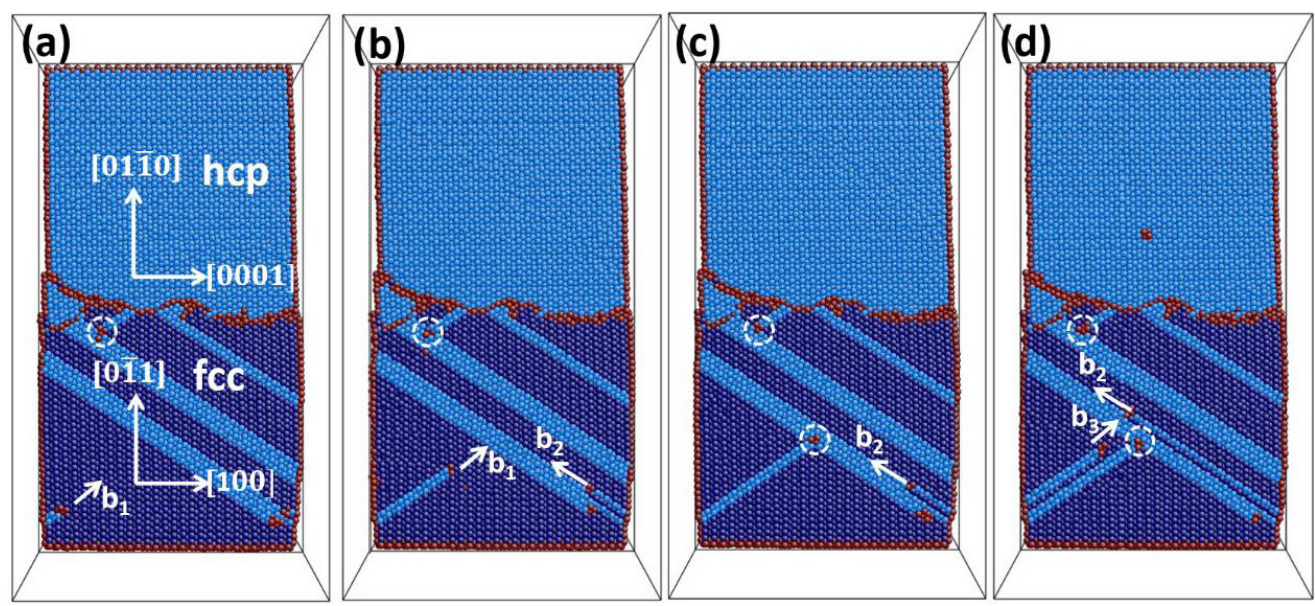

Figure 6 - Snapshots of partial dislocation nucleation and propagation from the HCP/FCC phase interfaces $\left(\mathbf{b}_{1}\right)$ and the corner $\left(\mathbf{b}_{2}\right)$ of nanopillars under compressive loading. The white dotted lines indicate the site where the partial dislocation is blocked by the HCP phase.

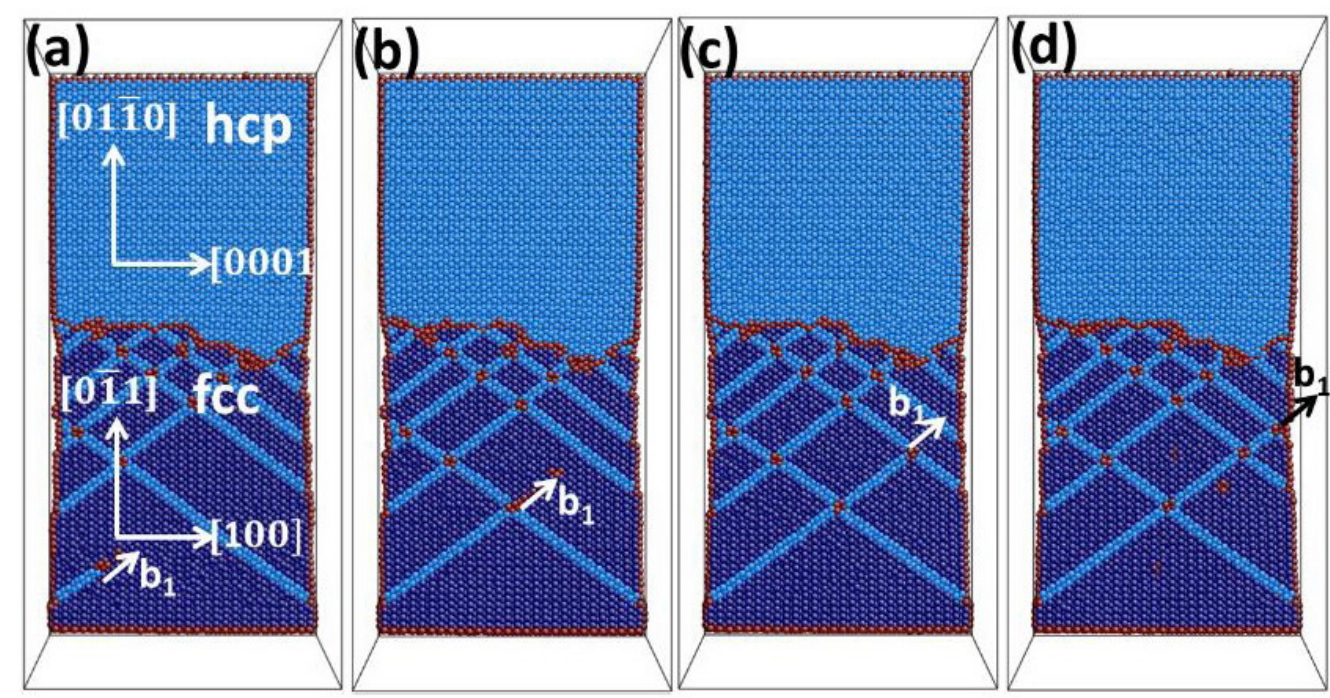

Figure 7 - Lomer-Cottrell sessile dislocation and network of stacking faults formed by Shockley partial dislocation reactions under tensile loading. 


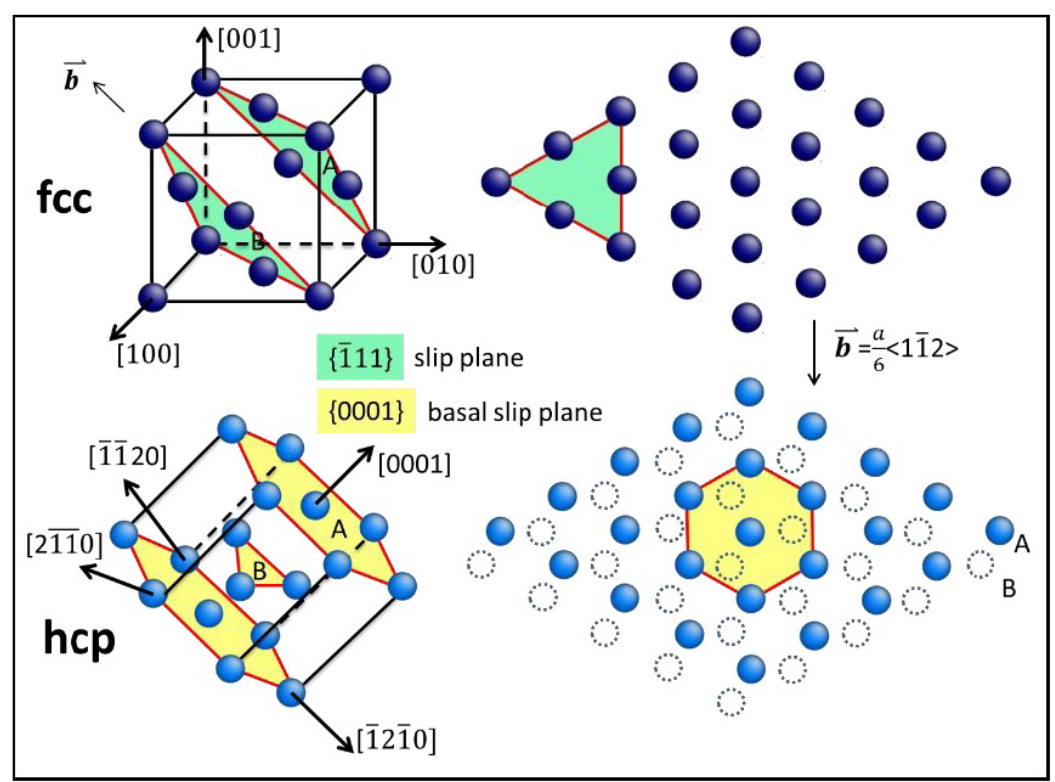

Figure 8 - Schematic illustration of direct phase formation from the metastable FCC phase to the HCP phase with the combination of Shockley partial edge dislocations and lattice distortion.

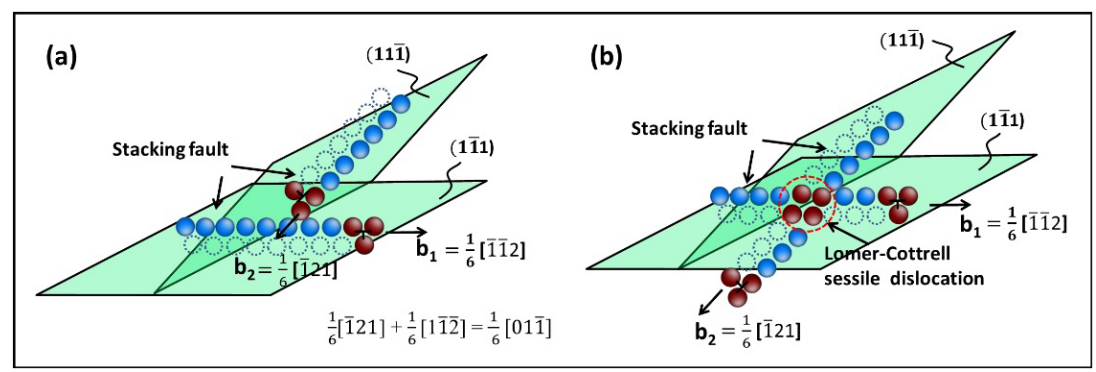

Figure 9 - Formation of a Lomer-Cottrell sessile dislocation.

nucleation location of Shockley partial dislocations increases obviously. It can be seen from Figure 2 that the tensile stress decreases at points $\mathrm{A}$ and $\mathrm{B}$, corresponding to the partial activation of the dislocation sources at the HCP/FCC interface of the nanopillar. A significant serrated-like behavior before point $\mathrm{C}$ is caused in the stress-strain curve of Figure $2 \mathrm{~b}$ due to the partial dislocation glide and dislocation reaction in the nanopillar. Atomic snapshots also confirm that a large number of Lomer-Cottrell sessile dislocations are generated before the maximum stress occurs. The HCP/FCC interface and free surfaces or corners of the nanopillars become favorable sites for dislocation nucleation. As shown in Figure 7a, a Shockley partial dislocation with Burgers vector $\mathbf{b}_{1}=\frac{1}{6}[\overline{1} 21]$ on the slip

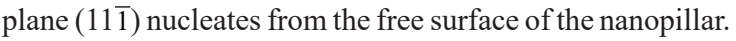
The dislocation clearly nucleates and grows on the surface or

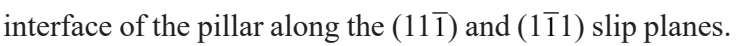
The Burgers vectors are determined to be $\frac{1}{6}[\overline{1} 21]$ and $\frac{1}{6}[1 \overline{12}]$ on the basis of the crystallographic analysis. These Shockley partial dislocations are accompanied by stacking faults. If the dislocations meet at the line of intersection of the (1111) and

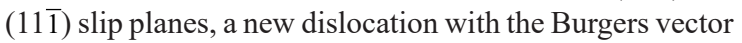
of $\frac{1}{6}[01 \overline{1}]$ is generated through the dislocation reaction, i.e a Lomer - Cottrell dislocation. The dislocation reaction can be expressed as

$$
\frac{1}{6}[\overline{1} 21]+\frac{1}{6}[1 \overline{12}]=\frac{1}{6}[01 \overline{1}]
$$

This dislocation reaction can be expressed by Thompson tetrahedron notation

$$
\overrightarrow{\alpha \mathrm{C}}+\overrightarrow{\mathrm{C} \beta} \rightarrow \overrightarrow{\alpha \beta}
$$

\subsection{Transformation mechanism of FCC phase to HCP phase in FCC crystal}

The transformation mechanism of the FCC phase to the HCP phase in the FCC crystal is illustrated in Figure 8. The unit cell of the FCC phase lies at the upper left angles of the diagram. The atomic plane shown in green in the FCC unit cell is the slip plane $\{\overline{1} 11\}$ which lies at upper right angles to the plane of the diagram. In the FCC unit cell the $\{\overline{1} 11\}$ layers are stacked in the sequence $\mathrm{ABCABC} \ldots$, and the lattice is perfect. Under compressive and tensile loading, the A layer atoms have slipped in the [1를 direction to a B layer position, the $\mathrm{B}$ atoms have slipped to $\mathrm{C}$, and the $\mathrm{C}$ atoms 
have slipped to A. This movement produces a stacking fault ABCBCABC... and a Shockley partial edge dislocation $\mathbf{b}_{1}=\frac{1}{6}[11 \overline{2}]$. The sequence of stacking faults conforms to the atomic sequence of the HCP structure, which liea at lower right angles to the plane of the diagram. The unit cell of the HCP phase liea at the lower left angles of the diagram. The atomic plane shown in yellow in the HCP unit cell is the slip plane $\{0001\}$. The phase transition from the metastable FCC phase to the HCP phase completed by Shockley partial edge dislocation nucleation, propagation, and accumulation. The lattice parameter of $0.4174 \mathrm{~nm}$ calculated for the FCC phase is almost the same as that reported earlier for 5-min deposited film $\left(0.41638 \mathrm{~nm}^{39}\right)$, mechanically milled polycrystalline Ti powder $\left(0.4237 \mathrm{~nm}^{40}\right.$ and $\left.0.4356 \mathrm{~nm}^{41}\right)$, Ti epitaxial films $\left(0.415 \mathrm{~nm}^{42}\right.$ and $\left.0.433 \mathrm{~nm}^{9}\right)$, and pure $\mathrm{Ti}$ after cryogenic channel-die compression deformation $\left(0.4302 \mathrm{~nm}^{17}\right)$. However, the result obtained from ab initio calculations $\left(0.411 \mathrm{~nm}^{20}\right)$ is smaller than the experimental values. Compared with the cohesive energy $\mathrm{Ecoh}=-4.85281 \mathrm{eV}$ for HCP phase Ti, the cohesive energy of the FCC phase $\left(\mathrm{E}_{\mathrm{coh}}=-4.839 \mathrm{eV}\right) \mathrm{Ti}$ is higher, which makes the HCP phase Ti more stable than the FCC phase $\mathrm{Ti}$ in the bulk. The lattice distortion amounts are $0 \%, 15.5 \%$ and $-10.8 \%$ along the $[11 \overline{2} 0],[1 \overline{1} 00]$ and [0001] directions, respectively. In the [1 $\overline{1} 00]$ direction, the atomic displacement of the lattice distortion is the largest.

The formation of a Lomer-Cottrell sessile dislocation is illustrated in Figure 9. The Lomer - Cottrell dislocation is a pure edge-type dislocation. The glide plane of the Lomer dislocation is (100), so it is sessile, i.e., the dislocation cannot glide on any of the $\{111\}$ glide plane. As the plastic deformation progresses, a large number of Lomer-Cottrell sessile dislocations and stacking fault nets are generated in the FCC phase of the nanopillar (Figure 7). These defects acs as a barrier to the glide of further dislocations on the two $\{111\}$ planes and are known as a Lomer- Cottrell lock. This also leads to a higher tensile stress (Figure 2), and maximum tensile stress is close to the compression stress, which could have an important effect on metal strain hardening and fracture.

\section{Conclusions}

Molecular dynamics simulations have been used to study the mechanical behavior and deformation mechanisms of Ti nanopillars containing an HCP/FCC phase boundary. The biphasic crystal yield is controlled by the nucleation and propagation of dislocations from the interface and free surface. Based on this study, the following conclusions can be drawn:

(1) The yield stress under tensile loading is much lower than that under compression, but has a similar maximum stress because a large number of Lomer-Cottrell sessile dislocations are produced during tensile loading.

(2) Plastic deformation occurs in the FCC phase and Shockley partial dislocation with Burgers vector $\frac{1}{6}<\overline{1} 21>$ are the primary deformation mode of the nanopillars during tensile and compressive loading.
The continuous accumulation of the deformation faults caused by the Shockley partial dislocations eventually leads to the FCC to HCP phase transformation in the FCC phase under compression. However, a large number of Lomer-Cottrell sessile dislocations and stacking fault nets are formed when the partial dislocations react during tensile loading, leading to an increase in tensile stress.

(3) The FCC phase can be easily transformed into the HCP phase, which is a metastable phase in biphasic nanopillars.

\section{Conflicts of interest}

The authors declare no conflicts of interest.

\section{Acknowledgements}

This project is financially supported by the National Key R\&D Program of China (Grant No. 2017YFA0700701, 2017YFA0700703), the Key projects to strengthen the foundation (YK180701), the National Natural Science Foundation of China (51701189, 51775055, 51601084 and 51662026), the Innovation ability improvement project of Gansu universities (2019A-028) and the Open fund of the State Key Laboratory of Advanced Processing and Recycling of Nonferrous Metals (SKLAB02019011).

\section{References}

1. Banerjee D, Williams J. Perspective on Titanium science and technology. Acta Mater. 2013;61(3):844-79.

2. Leyens $\mathrm{C}$, Peters M, editors. Titanium and titanium alloys: Fundamentals and applications. 2nd ed. Weinheim: Wiley-VCH; 2003.

3. Zong H, Lookman T, Ding X, Luo SN, Sun J. Anisotropic shock response of titanium: Reorientation and transformation mechanisms. Acta Mater. 2014;65(15):10-8.

4. Sikka SK, Vohra YK, Chidambaram R. Omega phase in materials Prog Mater Sci. 1982;27(3-4):245-310.

5. Singh AK, Mohan M, Divakar C. The kinetics of pressure-induced $\alpha \rightarrow \omega$ transformation in Ti. J Appl Phys. 1982;53(2):1221-3.

6. Todaka Y, Sasaki J, Moto T, Umemoto M. Bulk submicrocrystalline $\omega$-Ti produced by high-pressure torsion straining. Scr Mater. 2008;59(6):615-8.

7. Vohra YK, Spencer PT. Novel gamma-phase of titanium metal at megabar pressures. Phys Rev Lett. 2001;86(14):3068-71.

8. Yu Q, Kacher J, Gammer C, Traylor R, Samanta A, Yang Z, et al. In situ TEM observation of FCC Ti formation at elevated temperatures. Scr Mater. 2017;140:9-12.

9. Wawner FE Jr, Lawless KR. Epitaxial growth of titanium thin films. J Vac Sci Technol. 1969;6(4):588-90.

10. Chakraborty J, Kumar K, Ranjan R, Chowdhury SG, Singh SR. Thickness-dependent fcc-hcp phase transformation in polycrystalline titanium thin films. Acta Mater. 2011;59(7):261523.

11. Kim SK, Jona F, Marcus PM. Growth of face-centred-cubic titanium on aluminium. J Phys Condens Matter. 1996;8(1):253636.

12. Yamada Y, Yoshida K. Growth and structure of titanium evaporated films. Appl Sur Sci. 1988;33-34:465-71.

13. Yue LP, Yao WG, Qi ZZ, He YZ. Structure of nanometer-size crystalline Ti film. Nanostruct Mater. 1994;4(4):451-6.

14. Fazio M, Vega D, Kleiman A, Colombo D, Franco Arias LM, Márquez A. Study of the structure of titanium thin films deposited 
with a vacuum arc as a function of the thickness. Thin Solid Films. 2015;(593):110-5.

15. Chatterjee P, Sengupta SP. Evolution of dislocation substructure in vapour-deposited lead films on glass substrates. Philos Mag A. $2003 ; 83(8): 1033-44$.

16. Zhang DL, Ying DY. Formation of fec titanium during heating high energy ball milled Al-Ti powders. Mater Lett. 2002;50(2):149-53.

17. Jing R, Liang SX, Liu CY, Ma MZ, Zhang XY, Liu RP. Structure and mechanical properties of Ti-6Al-4V alloy after zirconium addition. Mater Sci Eng A. 2012;552(34):29-300.

18. Hong DH, Lee TW, Lim SH, Kim WY, Hwang SK. Stressinduced hexagonal close-packed to face-centered cubic phase transformation in commercial-purity titanium under cryogenic plane-strain compression. Scr Mater. 2013;69(5):405-8.

19. Xiong S, Qi W, Huang B, Wang M, Li Y. Size and shape dependent Gibbs free energy and phase stability of titanium and zirconium nanoparticles. Mater Chem Phys. 2010;120(2):446-51.

20. Sliwko VL, Mohn P, Schwarz K, Blaha P. The fcc - bec structural transition: I. A band theoretical study for $\mathrm{Li}, \mathrm{K}, \mathrm{Rb}, \mathrm{Ca}, \mathrm{Sr}$, and the transition metals Ti and V. J Phys Condens Matter. 1996;8(7):799-815.

21. Aguayo A, Murrieta G, Coss R. Elastic stability and electronic structure of fcc Ti, Zr, and Hf: A first-principles study. Phys Rev B. 2002;65(9):092106.

22. Ren J, Sun Q, Xiao L, Ding X, Sun J. Phase transformation behavior in titanium single-crystal nanopillars under [0001] orientation tension: A molecular dynamics simulation. Comput Mater Sci. 2014;9(25):8-12.

23. Ren J, Sun Q, Xiao L, Sun J. Temperature and strain rate effect of the deformation-induced phase transformation in pure titanium nanopillars oriented along [0001]. Comput Mater Sci. 2017;126:66-73.

24. Yang JX, Zhao HL, Gong HR, Song M, Ren QQ. Proposed mechanism of $\mathrm{HCP} \rightarrow \mathrm{FCC}$ phase transition in titianium through first principles calculation and experiments. Sci Rep. 2018;8(1):1992.

25. Zhao H, Hu X, Song M, Ni S. Mechanisms for deformation induced hexagonal close-packed structure to face-centered cubic structure transformation in zirconium. Scr Mater. 2017;132:63-7.

26. Nosé AS. molecular dynamics method for simulations in the canonical ensemble. Mol Phys. 1984;52(2):255-68.

27. Hoover WG. Canonical dynamics: equilibrium phase-space distributions. Phys Rev A Gen Phys. 1985;31(3):1695-7.

28. Plimpton SJ. Fast parallel algorithms for short-range molecular dynamics. J Comput Phys. 1995;117(1):1-19.
29. Li J. AtomEye: An efficient atomistic configuration viewer. Model Simul Mater Sci Eng. 2003;11(2):173-77.

30. Faken D, Jónsson H. Systematic analysis of local atomic structure combined with 3D computer graphics. Comput Mater Sci. 1994;2(2):279-86.

31. Tsuzuki H, Branicio PS, Rino JP. Structural characterization of deformed crystals by analysis of common atomic neighborhood. Comput Phys Commun. 2007;177(6):518-23.

32. Ackland GJ. Theoretical study of titanium surfaces and defects with a new many-body potential. Philos Mag A Phys Condens Matter Defects Mech Prop. 1992;66(6):917-32.

33. Ren J, Sun Q, Xiao L, Ding X, Sun J. Molecular dynamics simulations of the size effect of titanium single-crystal nanopillars orientated for double prismatic slips. Philos Mag Lett. 2013;10:583-90.

34. Ren J, Sun Q, Xiao L, Ding X, Sun J. Size-dependent of compression yield strength and deformation mechanism in titanium single-crystal nanopillars orientated [0001] and [1120]. Mater Sci Eng A. 2014;615:22-8.

35. Chang L, Zhou CY, Wen LL, Li J, He XH. Molecular dynamics study of strain rate effects on tensile behavior of single crystal titanium nanowire. Comput Mater Sci. 2017;128:348-58.

36. Chang L, Zhou CY, Pan XM, He XH. Size-dependent deformation mechanism transition in titanium nanowires under high strain rate tension. Mater Des. 2017;134:320-30.

37. Chang L, Zhou CY, Liu HX, Li J, He XH. Orientation and strain rate dependent tensile behavior of single crystal titanium nanowires by molecular dynamics simulations. J Mater Sci Technol. 2018;5:864-77.

38. Chang L, Zhou CY, Li J, He XH. Investigation on tensile properties of nanocrystalline titanium with ultra-small grain size. Comput Mater Sci. 2018;142:135-44.

39. Hirth JP, Lothe J. Theory of dislocations. New York: Wiley; 1982.

40. Chatterjee P, Gupta SPS. An X-ray diffraction study of strain localization and anisotropic dislocation contrast in nanocrystalline titanium. Philos Mag A Phys Condens Matter Struct Defects Mech Prop. 2001;81(1):49-60.

41. Sugawara Y, Shibata N, Hara S, Ikuhara Y. Interface structure of face-centered-cubic Ti thin film grown on $6 \mathrm{H}-\mathrm{SiC}$ substrate. J Mater Res. 2000;15(10):2121-4.

42. Kim SK, Marcus PM, Jona F. Growth of face-centred-cubic titanium on aluminium. J Phys Condens Matter. 1996;8(1):253636 . 